\title{
Idle Noise Reduction of a Parametric Acoustic Array Power Driver
}

\author{
Matija Pirc
}

\section{University of Ljubljana, Faculty of Electrical Engineering, Ljubljana, Slovenia}

\begin{abstract}
Parametric acoustic arrays (PAA) have progressed from specialized niche applications to commercially available audio solutions in the last two decades. Their primary advantage is their incredible directivity and their main disadvantage is low conversion efficiency of the primary ultrasonic waves into audible sound. This paper presents a noise analysis of a practical implementation of a directional audio system. The system is comprised of a modulator, a D-class audio amplifier, and an emitter consisting of 97 commercially available piezoelectric ultrasonic transducers. The designed system exhibited an uncomfortable level of idle noise at the maximum volume level. The analysis of the signal path and all the noise sources revealed that the most critical component was the modulator, and a solution was devised which provided a $16 \mathrm{~dB}$ improvement of the carrier to noise ratio.
\end{abstract}

Keywords: PAA, Parametric Acoustic Array, Directional Sound System, Ultrasonic, noise, PZT transducer

\section{Zmanjšanje lastnega šuma ojačevalnika in modulatorja za parametrično akustično polje}

\begin{abstract}
Izvleček: Parametrična akustična polja (PAP) so se v zadnjih dveh desetletjih razvila od specialnih nišnih aplikacij do komercialno dostopnih avdio sistemov. Njihova poglavitna prednost je izredna usmerjenost zvoka, glavna slabost pa nizka učinkovitost pretvorbe primarnega ultrazvočnega valovanja $v$ slišni zvok. $V$ tem prispevku je predstavljena analiza šuma praktične realizacije usmerjenega avdio sistema. Sistem sestavljajo modulator, avdio ojačevalnik razreda D in ultrazvočni oddajnik, sestavljen iz sedemindevetdesetih komercialno dostopnih piezoelektričnih ultrazvočnih pretvornikov. Zasnovani sistem je pri maksimalni nastavitvi glasnosti oddajal neprijetno visok nivo lastnega šuma. Analiza signalne poti in vseh možnih virov šuma je pokazala, da je najbolj kritična komponenta analogni množilnik. Na podlagi analize smo zasnovali rešitev, ki je izboljšala razmerje med velikostjo nosilca in šuma za 16 dB.
\end{abstract}

Ključne besede: PAP, parametrično akustično polje, usmerjeni zvočni sistem, ultrazvočni, šum, PZT pretvornik

*Corresponding Author's e-mail:matija.pirc@fe.uni-lj.si

\section{Introduction}

Parametric acoustic arrays (PAA) were first introduced by Westervelt in 1963 [1]. Due to their high directivity and almost non-existent side lobes, many applications have been developed using PAA ranging from underwater applications [2, 3], sediment exploration [4], communications [3, 5, 6], to medical applications [7]. In the last two decades, tremendous progress has been made in the field of PAAs in air to the point where there are multiple commercial solutions available for highly directive sound systems [8-10]. The two main challenges that each PAA system must overcome are the low conversion efficiency of the high frequency primary waves into secondary audible acoustic waves and the distortion of the desired signal. Since high ultrasound amplitudes are necessary to achieve the desired audio levels, noise can become a problem and care must be taken to keep it below the hearing threshold.

Conversion efficiency of the high frequency ultrasonic sound beam into audible sound is very low. To achieve sufficient volume of audible sound, the volume of the ultrasonic beam has to be very high and the signal source needs to be sufficiently powerful. The A and ABclass amplifiers are not very efficient, and therefore not well suited for this type of sound source. The D-class amplifiers with their high efficiency are a much better choice. The impedance of most ultrasonic transducers at resonant frequency has a very pronounced capacitive character, which requires a high reactive current in addition to the active current. The reactive current dramatically increases the losses of the linear power am- 
plifiers of both classes. D-class amplifiers employ pulse width modulation (PWM), nearly lossless switches, and an appropriate filter to achieve the same results as linear amplifiers, but with much lower losses. These amplifiers feature high efficiency for resistive, reactive or combined loads. On the other hand, D-class amplifiers can introduce noise into the system [11], especially if the PWM frequency is not considerably higher than the bandwidth of the amplified signal.

We designed our own directional sound system based on double sideband amplitude modulation (DSB-AM) using low cost ultrasonic piezoelectric ceramic transducers. The first version of the system exhibited quite high idle noise which was particularly noticeable at the maximum volume setting. Our initial noise analysis and measurements of the PAA power driver have already been presented in brief at the $50^{\text {th }}$ MIDEM conference [12]. In this paper an updated and more detailed account of the driver and its improvement is given. A short description of the operation principle of PAAs is followed by an overview of available ultrasonic transducers suitable for this purpose. Section 4 describes the electronics of the driver. Section 5 analyzes the noise performance of the electronics, and describes the changes made to the design. Measurement results and conclusions are given in the last two sections.

\section{Operation principle}

A parametric acoustic array is created by emitting an intense and highly directed ultrasonic beam consisting of a carrier wave and signal components introduced by one of the various modulation types [13]. The carrier interacts with other spectral components of the ultrasonic beam due to the nonlinearity of air at high acoustic sound pressure levels (SPL) and generates new spectral components along the ultrasonic beam.

These secondary waves contain audible spectral components related to the difference between the carrier and side bands. The nonlinearity of air also generates higher inaudible frequencies which are quickly absorbed due to the high absorption coefficient of air at high frequencies. Thus the ultrasonic source creates

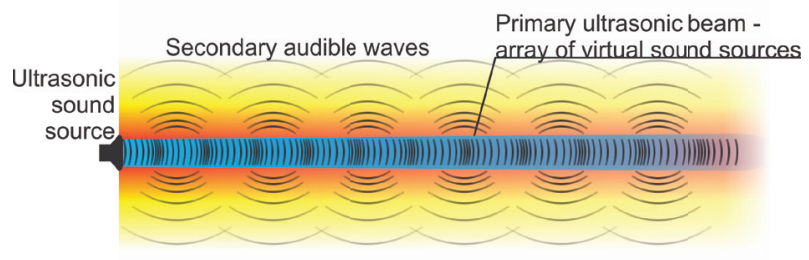

Figure 1: Visualization of the PAA. a beam of ultrasound which acts as a virtual array of sound sources (Fig. 1).

Berktay devised a simple equation in 1965 [6] to describe the time dependent pressure of low-frequency secondary waves in the far-field

$p\left(t^{\prime}\right)=p_{0}^{2} \frac{\beta a^{2}}{16 \rho_{0} c_{0}^{4} z \alpha_{0}} \cdot \frac{d^{2}}{d t^{\prime 2}} E^{2}\left(t^{\prime}\right)$

where $E\left(t^{\prime}\right)$ is the envelope of the modulated ultrasonic sound pressure, $p_{0}$ is the pressure source amplitude, $\beta$ is the nonlinearity of the medium, which is 1.2 for air, $a$ is the source radius, $\rho_{0}$ is the medium density, $c_{0}$ is the speed of sound in the medium, $z$ is the distance from the source along the axis of the beam, $a_{0}$ is the sound absorption coefficient of the medium for the carrier frequency, and $t^{\prime}$ is retarded time

$t^{\prime}=t-z / c_{0}$

Ten years later Merklinger [14] upgraded Berktay's expression to account for nonlinear attenuation at higher ultrasound intensities. For SPL of the primary beam exceeding $120 \mathrm{~dB}$, with $0 \mathrm{~dB}=20 \mu \mathrm{Pa}$, Merklinger's relation can be linearized to

$$
p_{s}\left(t^{\prime}\right) \propto p_{0} \frac{d^{2}}{d t^{2}}\left|E\left(t^{\prime}\right)\right|
$$

whereby the less important constants have been omitted for the sake of clarity. According to (3) the level of secondary sound becomes linearly proportional to the second time-derivative of the envelope of the modulated ultrasound carrier. This linearized relation in combination with the simplicity of design is the reason that simple double sideband amplitude modulation (DSB$A M$ ) is frequently used in PAAs for audio reproduction.

We can report that the perceived quality of sound reproduction we achieved using the DSB-AM was much better than expression (1) and the work of Yoneyama [15] would suggest. However, in the case of lower primary wave intensities the perceived sound is noticeably impaired, requiring nonlinear preprocessing of the envelope to compensate for the squaring in (1) as suggested in $[13,15]$. Hearing tests using a computer generated square rooted envelope of speech and music demonstrated substantially improved quality of sound reproduction at the lower ultrasonic carrier levels. The designed driver is intended for louder reproduction, which requires a high ultrasound level of the carrier (SPL $\approx 120 \mathrm{~dB}$ ), therefore simple DSB-AM is employed to minimize costs and the physical dimensions of the device. The high SPL of the carrier ensures that the airborne demodulation process is ruled by Merkliger's relation (3), which does not include the square of the 
envelope waveform, and therefore does not cause high total harmonic distortion (THD).

\section{Ultrasonic sound source}

Ultrasonic sound waves in the air are usually generated by piezoelectric or electrostatic transducers. The commercially available electrostatic transducers we tested were not suitable for PAAs. Electrostatic transducers require high driving voltages with a high DC bias. The tested transducer achieved an SPL of $115 \mathrm{~dB}$ at 50 $\mathrm{kHz}$ at the highest combined voltage of 300Vpk-pk + 100 VDC.

The advantages of electrostatic transducers with respect to other types of transducers are wide bandwidth, insensitivity to overload, and a narrow beam angle. The drawbacks are high driving voltage and DC bias, their high price, and the small number of manufacturers.

Piezoelectric transducers use piezo ceramics or piezoelectric polymers in the form of thin foils. Only the ceramic transducers are commercially available, so the power amplifier has been designed for driving a variable number of commercially available piezoelectric transducers.

The mechanical construction of a piezoelectric ceramic transducer is shown in Fig. 2. The active element is a bimorph of Lead Zirconate Titanate (PZT) ceramics. The two PZT layers are poled differently [16], so that the applied voltage causes one layer to shrink while the other expands. The center of the bimorph disc is thus displaced vertically up or down depending on the polarity of the voltage. The mechanical properties of the materials used, as well as their physical dimensions, determine the resonant frequency and bandwidth of the transducer.
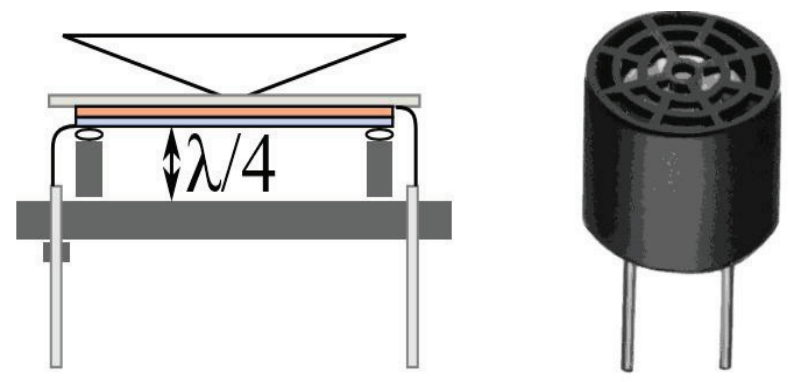

Figure 2: Cross-section and appearance of the utilized PZT bimorph transducer.

These transducers can continuously transmit an SPL of about $120 \mathrm{~dB}$ at $40 \mathrm{kHz}$ when driven by $10 \mathrm{~V}_{\mathrm{RMS}}$. The PZT bimorphs we used have a small bandwidth and a maximum driving voltage of $20 \mathrm{~V}_{\text {RMS }}$. Internal losses cause heat and mechanical fatigue which irreversibly destroy the weakest part of the transducer, namely the joint between the top metal plate and the acoustic cone. When the joint breaks, the light metal cone becomes loosened and the acoustic coupling between the transmitter and the air is lost. Such destruction alters the impedance at the electrical terminals of the transducer which can lead to an increase of the driving voltage if resonant filtering is used.

Both electrostatic and piezoelectric polymer film transducers are less sensitive to overloading and have a greater bandwidth than PZT bimorphs, but require high driving voltages. In addition, commercially available electrostatic transducers do not achieve the desired acoustic pressure level required for PAAs unless they are driven with very high voltages of $400 \mathrm{~V}_{\mathrm{pp}}$. Piezoelectric film transducers require lower driving voltages - typically about $100 \mathrm{~V}_{\mathrm{RMS}}$ - to achieve a sufficient SPL of the ultrasonic carrier, but are not available as commercial components.

\subsection{PZT bimorph electrical model}

The structure of the PZT transducer shown in Fig. 2 is similar to the structure of a quartz crystal oscillator. Metallic electrodes on both surfaces of the PZT ceramics with very high permittivity $\varepsilon_{r}$ form a physical capacitor $C_{p}$ between the terminals. The electromechanical reaction of the piezoelectric disc, material losses and transmitted acoustic power are modeled by a serial RLC network. The electrical equivalent network of piezoelectric transducer is shown in Fig. 3. The parallel capacitance $C_{p}$ is measured between the terminals at $1 \mathrm{kHz}$ and is usually specified by the manufacturer along with other relevant data. The plot in Fig. 4 shows the measurement of the complex admittance $Y(f)$ of the PZT transducer used in the ultrasonic transmitter. The trajectory in the complex plane was measured with a HP 3589A spec-

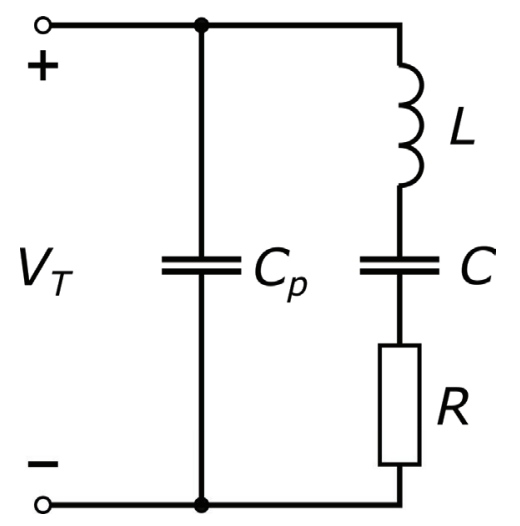

Figure 3: Equivalent electrical model of the PZT ceramic transducer 
trum/network analyzer and an additional linear power amplifier which provided the required driving voltage of $10 \mathrm{~V}_{\mathrm{RMS}}$. Two sets of element values of the transducer electrical model (Fig. 3) are shown in Table 1. One set is obtained from the complex impedance plot from the datasheet [17], which was acquired using a test voltage of $1 \mathrm{~V}_{\mathrm{RMS}}$, the other is calculated from the measured plot shown in Fig. 4.

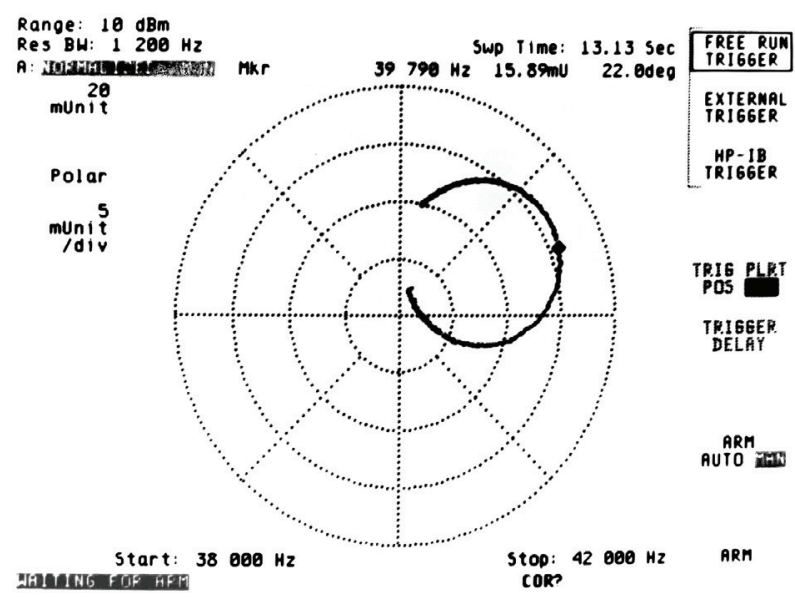

Figure 4: Vector plot of the transducer admittance $Y(f)$ from $38 \mathrm{kHz}$ to $42 \mathrm{kHz}\left(V_{T}=10 \mathrm{~V}_{\mathrm{RMS}}\right)$

Table 1: Equivalent circuit element values for Pro-Wave 400ST16P

\begin{tabular}{|c|c|c|c|c|c|}
\hline Data source & $\mathrm{V}_{\mathrm{T}}$ & $\mathrm{C}_{\mathrm{p}}[\mathrm{nF}]$ & $\mathrm{R}[\Omega]$ & $\mathrm{L}[\mathrm{mH}]$ & $\mathrm{C}[\mathrm{pF}]$ \\
\hline Data sheet & $1 \mathrm{~V}_{\text {RMS }}$ & 2.4 & 760 & 93 & 167 \\
\hline Measurement & $10 \mathrm{~V}_{\text {RMS }}$ & 1.9 & 665 & 81 & 197 \\
\hline
\end{tabular}

Slight differences between the values are expected and come from device tolerances and different driving voltages. The actual operating point of the transmitter varies between 0 and $10 V_{R M S^{\prime}}$ depending on the volume setting. The ultrasonic transmitter of the de-

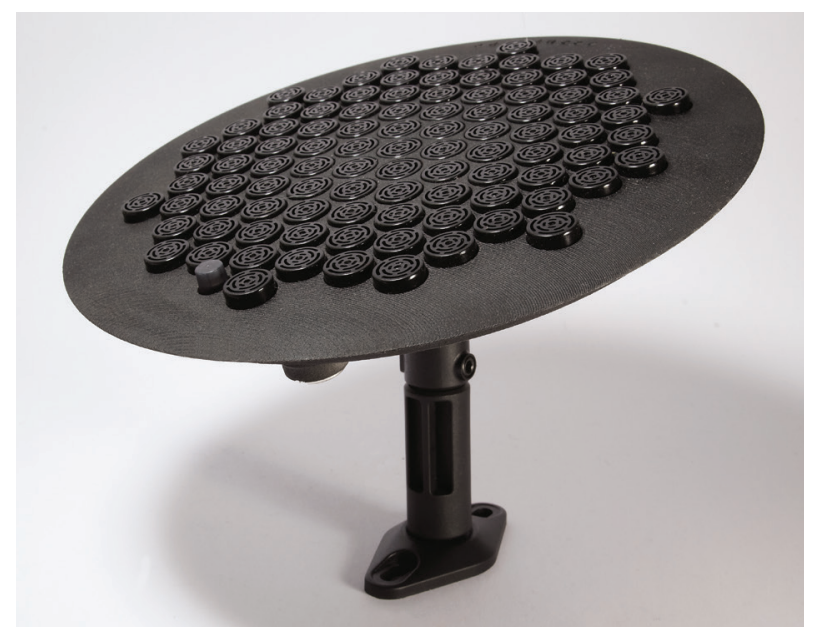

Figure 5: Photograph of the PZT ultrasonic transmitter signed system shown in Fig 5 consists of 97 400ST16P piezoelectric transducers (Pro-Wave El. Corp.) arranged in a circle.

\section{Modulator/amplifier}

The modulator/amplifier system consists of (Fig. 6):

- Input amplifier and signal conditioner,

- Low THD 40 kHz quadrature oscillator,

- Integrated analogue multiplier with an adder,

- $\quad$ Differential output amplifier with a DC blocking filter,

- D-class power amplifier,

- $\quad$ LC output filter, and

- $\quad$ Power supply (not shown).

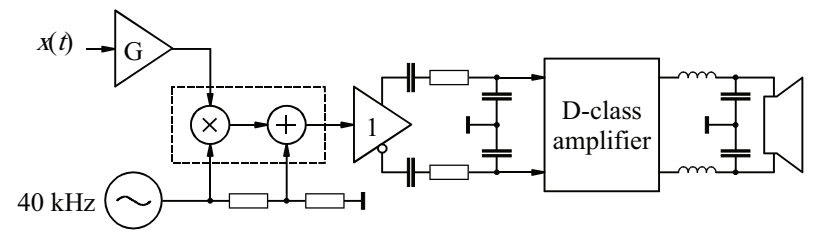

Figure 6: Main building blocks of the modulator/amplifier system

The amplitude of the input signal $x(t)$ is adjusted within the frequency range between $50 \mathrm{~Hz}$ and $20 \mathrm{kHz}$, by a two-stage preamplifier, shown as an amplifier with gain $\mathrm{G}$ in Fig. 6. If a stereophonic sound source is applied at the driver input, the two signals are added together in the first stage of the preamplifier. The ultrasonic carrier frequency is generated by a quadrature oscillator consisting of two low-noise operational amplifiers. This is a low cost solution with stable frequency that can be adjusted by a trimmer. The frequency drift of the $40 \mathrm{kHz}$ carrier over the temperature interval $\pm 30^{\circ} \mathrm{C}$ is within $\pm 150 \mathrm{~Hz}$, which is much less than the specified center frequency tolerance $\Delta f_{0}= \pm 1 \mathrm{kHz}$ of the selected PZT transducers. Amplitude stabilization is implemented according to the guide lines [18] for low harmonic distortion. The higher harmonic components lie far outside of the transmitting frequency band around the carrier but nonlinearity of the multiplier and action of switch mode power amplifier can generate intermodulation components in the AM signal frequency band.

The double sided AM which has proven to be suitable for PZT transducers is implemented by an AD633, which contains an analog four quadrant multiplier and an adder. The AM signal is obtained by adding the DSB modulated signal, which appears at the multiplier output to the carrier

$$
y(t)=A \cos \omega_{c} t[1+m x(t)]
$$


where $A$ is the carrier amplitude and $m$ is the modulation index. The expression in brackets is the envelope function $E(t)$, which is proportional to the instantaneous carrier amplitude.

A D-class power amplifier is used to avoid large heat sinks and the demanding power supply required by linear power amplifiers. The complex admittance $Y\left(\omega_{c}\right)$ of the transmitter at the carrier frequency, which is also the resonant frequency of PZT transducers, can be approximated by

$$
Y\left(\omega_{C}\right)=\frac{n}{R}+j \omega_{C} n C_{p}
$$

where $R$ and $C_{p}$ are the model parameters of Fig. 3 given in Table 1, while $n$ is the number of transducers in the transmitter. The actual admittance of the load for $n=97$ transducers at the carrier frequency of $40 \mathrm{kHz}$ is $Y\left(\omega_{c}\right)=(146+j 46) \mathrm{mS}$, which corresponds to an impedance of $6.53 \Omega<-17.7^{\circ}$. This means that the current through the resistance, which reflects the acoustic load, is accompanied by the reactive current through the parallel capacitance of the transducer electrodes. This current, whose magnitude is roughly a third of the current through the acoustic load, has to be delivered by the output stage. If an AB-class amplifier would be used, it would dissipate $18 \mathrm{~W}$ just for the $10 \mathrm{~V}_{\text {RMS }}$ carrier at the output. This calculation was made for the supply voltages of $\pm 24 \mathrm{~V}$ which would be required for achieving the maximum allowed transmitter voltage of $20 \mathrm{~V}_{\text {RMS }}$ at $100 \%$ modulation.

D-class amplifiers are much more efficient than linear amplifiers, which usually operate in an AB-class, but require a low-pass output filter to suppress the spectral components repeated around multiples of the operating PWM frequency $f_{P W M}$. In the case of more usual applications, where the maximum signal frequency is $20 \mathrm{kHz}$, the PWM switching frequency of only $200 \mathrm{kHz}$ in combination with an appropriate low pass filter could suffice. The highest frequency of the AM signal in this case is $60 \mathrm{kHz}$, which requires a higher switching frequency to achieve sufficient frequency separation between the base band and the first lower side band of the PWM signal.

In the implemented driver, a TAS5613A integrated switching amplifier with $f_{P W M}=400 \mathrm{kHz}$ was used. The filter for the bridge tied load (BTL) topology is symmetrical with respect to ground in order to reduce the electromagnetic interference emissions (EMI) from the transmitter leads.

A simplified asymmetrical model of the output filter and the equivalent electrical model of the ultrasonic transmitter are shown in Fig. 7, where a clear distinc- tion between the filter capacitors inside the driver and the transmitter model is made. The plot in Fig. 8 shows the electrical and acoustic frequency response, reflecting the voltage across the terminals and the resistor voltage, respectively.

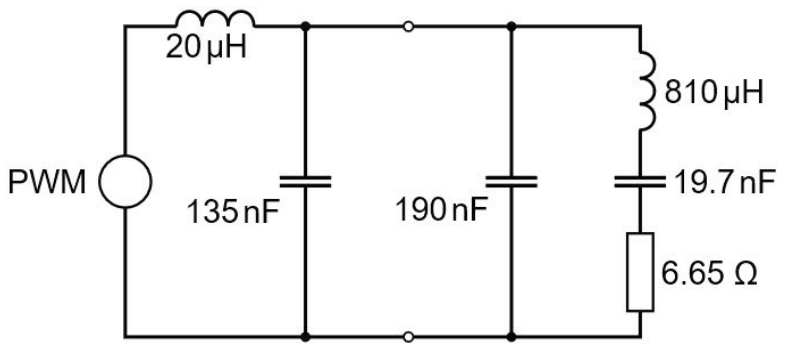

Figure 7: The output filter connected to the equivalent electrical model of the transmitter.

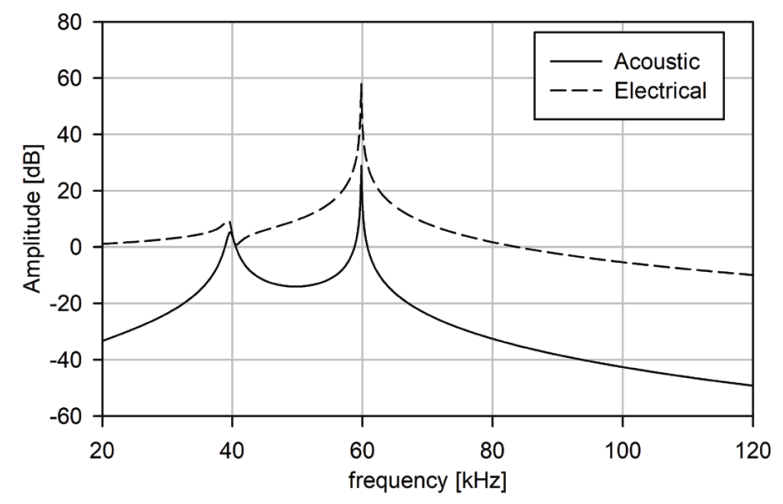

Figure 8: Frequency response of the loaded output filter.

Due to the capacitive nature of the load determined by $C_{p}$ the frequency response $|H(f)|$ of the filtering network has a second resonant peak in the spectral region above the base-band of the AM signal, which spans from $20 \mathrm{kHz}$ to $60 \mathrm{kHz}$, and below the lower margin of the first mirror band at $340 \mathrm{kHz}$. This part of the spectrum does not contain any PWM spectral components. However, the shape of the acoustic transfer function modifies the noise power spectrum within the pass band of the signal and has an influence on the audible noise.

The BTL configuration of the switching amplifier, which doubles the output voltage swing compared to a single ended configuration, is achieved by inverting the signal at the input of one of the two amplifiers of the switching power driver. The differential output signals are AC-coupled to the switching amplifier as shown in Fig. 6. The inputs of the amplifiers are internally biased and the DC level is adequate providing there is no DC path to the input node. The network shown in the schematics performs much better than a coupling capacitor alone. The resistor in the signal path limits the input 
current and adds a real pole in the frequency response of the coupling.

\section{Noise source analysis}

\subsection{Noise analysis of the original system}

The suitability of a simple AM and PZT bimorphs for a PAA directional sound system was verified by the hearing tests conducted on the system. The flaws of AM in PAAs mentioned in $[13,16]$ were not perceived for different audio signals, i.e., music, speech, etc., although high idle noise levels were noticed. Acoustic noise measurements in this particular case would be extremely demanding, because of the very large dynamic range required due to the very high ultrasonic carrier signal. The normal hearing SPL is roughly 40 to $50 \mathrm{~dB}$ below the ultrasonic carrier level and the perceivable noise SPL is estimated to be about $40 \mathrm{~dB}$ lower than that, depending on the background noise of the surroundings.

The noise spectrum range $\left(f_{C}-f_{H^{\prime}} f_{C}+f_{H}\right)$ modulates the carrier amplitude and thus demodulates as audible noise. As a measurable figure of merit we use the ratio between the power of the carrier and the noise, given by

$$
C N R=10 \log \frac{C}{N}=10 \log \frac{A^{2}}{2 \cdot S_{n} B}
$$

where $C$ is the carrier power, $N$ is the in band noise power, $A$ is the carrier amplitude, $S_{n}$ is noise power spectral density, and $B$ is the frequency bandwidth.

Within the PAA driver system (Fig. 6) each functional block generates noise that contributes to the final CNR at the transmitter. The two low noise JFET operational amplifiers TL071 used in the two-stage input preamplifier have an equivalent input noise of $4 \mu \mathrm{V}$ from $100 \mathrm{~Hz}$ to $10 \mathrm{kHz}$ and a constant noise density of $18 \mathrm{nV} / \sqrt{\mathrm{Hz}}$ above $10 \mathrm{kHz}$. The contribution of the input noise current is negligible and is not taken into account.

Each of the two stages of the input preamplifier can be represented by the equivalent circuit shown in Fig. 9, where all the noise sources are joined into one equivalent input noise source. The equivalent input noise voltage consists of the amplifiers input noise and all the thermal noise sources:

$u_{n e q}=\sqrt{u_{n T L 071}^{2}+u_{n R}^{2}}$

where $u_{n T L 071}$ represents input noise of the operational amplifier and $u_{n R}$ all the thermal noise contributions. Contributions of all the thermal noise sources can be modeled by a single thermal noise source with noise voltage appropriate to the parallel connection of resistors $R_{1}$ and $R_{2}$ of Fig. 9:

$u_{n R}=\sqrt{4 k B T \frac{R_{1} R_{2}}{R_{1}+R_{2}}}$

where $k$ represents Boltzmann constant, $B$ bandwidth and $T$ temperature. Given the equivalent circuit in Fig. 9, the output noise can be calculated as:

$u_{\text {nout }}=u_{\text {neq }} \frac{R_{1}+R_{2}}{R_{1}}$

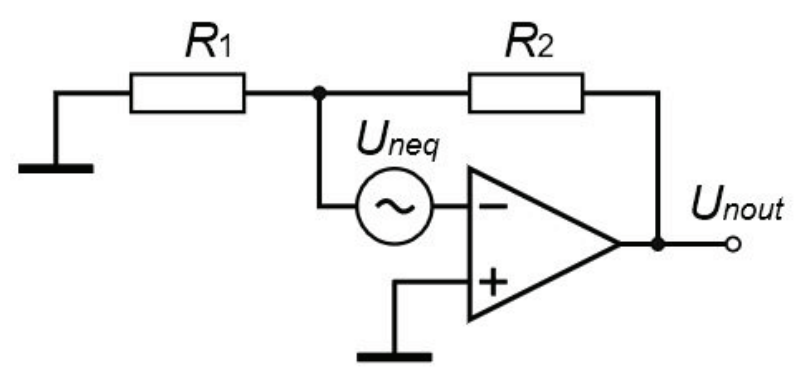

Figure 9: Equivalent circuit applicable to both stages of the input preamplifier.

The noise voltages and relevant data for the two-stage input audio signal conditioner are shown in Table 2.

Table 2: Input amplifier noise voltages $(B=20 \mathrm{kHz}, T$ $=300 \mathrm{~K})$.

\begin{tabular}{|l|l|l|} 
Stage & 1 & 2 \\
\hline $\mathrm{R}_{1}$ & $5.0 \mathrm{k} \Omega$ & $10.0 \mathrm{k} \Omega$ \\
\hline $\mathrm{R}_{2}$ & $4.7 \mathrm{k} \Omega$ & $10.0 \mathrm{k} \Omega$ \\
\hline Thermal input noise & $0.9 \mu \mathrm{V}$ & $1.3 \mu \mathrm{V}$ \\
\hline Amplifier input noise & $4.4 \mu \mathrm{V}$ & $4.4 \mu \mathrm{V}$ \\
\hline Equivalent input noise & $4.5 \mu \mathrm{V}$ & $4.9 \mu \mathrm{V}$ \\
\hline Inverting gain & 0.94 & 1 \\
\hline Output noise & $8.7 \mu \mathrm{V}$ & $9.2 \mu \mathrm{V}$ \\
\hline Entire output noise & \multicolumn{2}{|c|}{$12.7 \mu \mathrm{V}$} \\
\hline
\end{tabular}

The most important noise source in this spectral region is the $1 / \mathrm{f}$ noise of the amplifier. Thermal noise is not significant because of the small bandwidth and low input resistances.

The oscillator generates the carrier signal $(40 \mathrm{kHz}$, $3.3 \mathrm{~V}_{\mathrm{RMS}}$ ) and noise with significant noise density in the vicinity of the carrier:

$c(t)=s_{C}(t)+n_{C}(t)$ 
where $n_{c}(t)$ is the carrier signal noise and $s_{c}(t)$ is the ideal carrier signal:

$$
s_{C}(t)=A \cdot \cos \left(\omega_{C} t\right)
$$

The total noise power must be integrated over the $40 \mathrm{kHz}$ band - carrier frequency $f_{c} \pm 20 \mathrm{kHz}$. An equivalent noise voltage of $96 \mu \mathrm{V}$ was obtained using LTspice and operational amplifier models provided by the manufacturer. This gives a CNR of $91 \mathrm{~dB}$ at the oscillator output. The result does not include harmonic components because they are outside the frequency range of interest.

The carrier and audio signal are multiplied together and divided by $10 \mathrm{~V}$ by the AD633 integrated circuit. The carrier signal is attenuated by a factor of 10 by a resistor divider and added to the product:

$y(t)=\frac{x(t) \cdot c(t)}{10 \mathrm{~V}}+\frac{c(t)}{10}$

Both the carrier and the signal each contain their own noise and the multiplication product from (12) contains multiple terms representing noise:

$$
\begin{aligned}
x \cdot c & =\left(s_{X}+n_{X}\right) \cdot\left(s_{C}+n_{C}\right)= \\
& =s_{X} s_{C}+s_{X} n_{C}+n_{X} s_{C}+n_{X} n_{C}
\end{aligned}
$$

where $s_{X}$ is the ideal input signal, $n_{x}$ is the signal noise, $s_{c}$ is the ideal carrier signal, and $n_{c}$ is the carrier noise. The term $s_{X} s_{C}$ is the desired signal, while all other terms represent noise. The product of both noises $n_{x} n_{c}$ is very small and can be neglected. When idle noise is considered, the signal is zero and therefore the product of the carrier noise with the signal $s_{x} n_{c}$ is also zero. The only term which contributes significantly to the idle noise at the output of the multiplier is the signal noise multiplied by the carrier signal $n_{x} s_{c}$, which scales and spreads the carrier noise over the pass-band frequency range $f_{c} \pm 20 \mathrm{kHz}$. Taking into account the scaling factor of $1 /(10 \mathrm{~V})$ it contributes:

$$
n_{D S B}=\frac{3.3 \mathrm{~V} \cdot 12.7 \mu \mathrm{V}}{10 \mathrm{~V}}=4.2 \mu \mathrm{V}
$$

After the multiplication of the signal with the carrier, the carrier signal attenuated by a factor of 10 is added, which contributes its own noise of $9.6 \mu \mathrm{V}$ to the output signal. The AD633 itself generates noise with a constant spectral density of $0.8 \mu \mathrm{V} / \sqrt{\mathrm{Hz}}$ which results in $160 \mu \mathrm{V}$ of noise within the $40 \mathrm{kHz}$ bandwidth of interest. Particular noise source contributions, the entire output noise and the obtained CNR are summarized in Table 3.
Table 3: Multiplier noise sources $(B=40 \mathrm{kHz})$.

\begin{tabular}{|l|l|}
\hline Multiplier output noise & $4.2 \mu \mathrm{V}$ \\
\hline $0.33 \mathrm{~V}$ carrier noise & $9.6 \mu \mathrm{V}$ \\
\hline AD633 output noise & $160 \mu \mathrm{V}$ \\
\hline Entire output noise & $160.3 \mu \mathrm{V}$ \\
\hline CNR & $66.3 \mathrm{~dB}$ \\
\hline
\end{tabular}

The differential output amplifier noise contributes a similar amount of noise as the first stage of the input amplifier (approximately $5 \mu \mathrm{V}$ ). Compared to the noise present in the signal at this stage it is insignificant and can be safely neglected.

The carrier and accompanying noise are amplified and additional uncorrelated noise is generated by the Dclass TAS5613A power amplifier. The gain of the amplifier for a single channel is 22.4, but in the BTL configuration used in this case, the gain is 44.8. The amplitude of the carrier that appears on the transmitter in idle state at full volume is calculated to be $7.4 \mathrm{~V}_{\mathrm{RMS}}$, but in reality varies with temperature between approximately 7 and 8.5 $\mathrm{V}_{\text {RMS }}$. Taking into account the transmitter impedance at $40 \mathrm{kHz}$, the calculated carrier voltage results in an apparent output power of 8.4 VA and effective output power of $8.0 \mathrm{~W}$. This level was selected to ensure that the driving voltage never exceeds the limits of safe operation of the power amplifier and the piezoelectric transducers.

The only information the TAS5613 datasheet contains about noise is a THD+N (Total Harmonic Distortion and Noise) parameter expressed in percent. This means the noise and THD at the output depend on signal size. In this specific case, harmonic distortion is not important because the harmonic components lie outside the pass-band of the AM signal. It is possible to separate the THD and $\mathrm{N}$ portions of the THD+N parameter with the help of the datasheet. The THD+N parameter drops significantly above $10 \mathrm{kHz}$ because the higher harmonic components of signals with a frequency above $10 \mathrm{kHz}$ lie beyond the audio band. That means that for signals above $10 \mathrm{kHz}$ only noise contributes to the THD+N parameter (Table 4).

By means of careful measurements a third kind of output noise was identified at the output of the power amplifier with the inputs AC-shorted by capacitors. This noise, which is not specified in the data sheet, was measured on the transmitter in the frequency range from $20 \mathrm{kHz}$ to $60 \mathrm{kHz}$. Table 4 summarizes all the relevant parameters, noise contributions, final output noise and CNR at the transmitter terminals. 
Table 4: Power amplifier noise voltages $V_{o}=C=7.4 \mathrm{~V}_{\text {RMS }}$ $P_{L}=8.0 \mathrm{~W}$

\begin{tabular}{|l|l|}
\hline THD $+\mathrm{N}(\mathrm{f}>10 \mathrm{kHz})$ & $0.002 \%=2 \cdot 10^{-5}$ \\
\hline Voltage gain & $2 \times 22.4$ \\
\hline Amplified input noise & $3.60 \mathrm{mV}$ \\
\hline Idle output noise (input shorted) & $0.49 \mathrm{mV}$ \\
\hline Generated output noise (carrier) & $0.15 \mathrm{mV}$ \\
\hline Entire output noise & $3.63 \mathrm{mV}$ \\
\hline CNR & $66.2 \mathrm{~dB}$ \\
\hline
\end{tabular}

The presented analysis of the sources and propagation of noise reveals that the most important source of noise is the multiplier. The data in Table 4 shows that the noise generated by the power stage is negligible in relation to the amplified input noise. The input and output signals have almost the same CNR.

The noise generated by each stage (except the power amplifier stage) is independent of the signal size unless the limits of a stage are exceeded. A general solution to the signal to noise ratio problem is to either decrease the noise or increase the signal amplitude. In this case, the most critical stage regarding generated noise is the AD633 multiplier. There is considerable headroom available with regard to signal size at this stage, while nothing can be done to reduce the noise generated by the AD633. Therefore, the solution would be to increase signal size before it is passed through the multiplier and reduced again just before the power amplifier stage.

\subsection{Noise analysis of the improved system}

In order to increase the CNR at the output of the multiplier, the voltage gain of the second stage of the input amplifier was increased from 1 to 10 and the oscillator output was connected to the summing input of the AD633 directly, without any attenuation to maintain the same modulation depth of the AM signal. The amplitudes of all signals involved are still below the saturation margins of the device. The multiplier output had to be attenuated by approximately a factor of 10 before it could be fed into the power amplifier in order to maintain the same signal levels as before. To achieve the attenuation, the capacitances of both DC blocking networks at the power amplifier input were changed. Due to the sparse value scales of the multilayer ceramic capacitors the capacitive voltage divider attenuates the output voltage to $13 \%$ instead of $10 \%$ in the pass band between $1 \mathrm{kHz}$ and $1 \mathrm{MHz}$.

Had the multiplier been the only source of noise, this modification would have resulted in a $20 \mathrm{~dB}$ improvement of the CNR. Tables 5, 6 and 7 present the noise magnitudes for the input stage, the modulator and the power amplifier, respectively, of the improved system.

The results show that the CNR at the output of the modulator is indeed improved by $18.5 \mathrm{~dB}$, however the CNR at the output of the power amplifier is slightly less improved, namely, by only $15.9 \mathrm{~dB}$, since the noise generated by the power amplifier is not negligible anymore.

Table 5: Modified input amplifier noise voltages $(B=20$ $\mathrm{kHz}, \mathrm{T}=300 \mathrm{~K})$.

\begin{tabular}{|l|l|l|} 
Stage & 1 & 2 \\
\hline $\mathrm{R}_{1}$ & $5.0 \mathrm{k} \Omega$ & $1.0 \mathrm{k} \Omega$ \\
\hline $\mathrm{R}_{2}$ & $4.7 \mathrm{k} \Omega$ & $10.0 \mathrm{k} \Omega$ \\
\hline Thermal input noise & $0.9 \mu \mathrm{V}$ & $0.55 \mu \mathrm{V}$ \\
\hline Amplifier input noise & $4.4 \mu \mathrm{V}$ & $4.4 \mu \mathrm{V}$ \\
\hline Equivalent input noise & $4.5 \mu \mathrm{V}$ & $4.4 \mu \mathrm{V}$ \\
\hline Inverting gain & 0.94 & 10 \\
\hline Output noise & $8.7 \mu \mathrm{V}$ & $48.4 \mu \mathrm{V}$ \\
\hline Entire output noise & \multicolumn{2}{|c|}{$99.6 \mu \mathrm{V}$} \\
\hline
\end{tabular}

Table 6: Multiplier noise sources ( $B=40 \mathrm{kHz}$ ).

\begin{tabular}{|l|l|}
\hline Multiplier output noise & $32.9 \mu \mathrm{V}$ \\
\hline $3.3 \mathrm{~V}$ carrier noise & $96 \mu \mathrm{V}$ \\
\hline AD633 output noise & $160 \mu \mathrm{V}$ \\
\hline Entire output noise & $189 \mu \mathrm{V}$ \\
\hline CNR & $84.8 \mathrm{~dB}$ \\
\hline
\end{tabular}

Table 7: Power amplifier noise voltages $V_{o}=C=9.7 \mathrm{~V}_{\mathrm{RMS}^{\prime}}$ $P_{L}=13.7 \mathrm{~W}$.

\begin{tabular}{|l|l|}
\hline Amplified input noise & $0.55 \mathrm{mV}$ \\
\hline Idle output noise (input shorted) & $0.49 \mathrm{mV}$ \\
\hline Generated output noise (carrier) & $0.19 \mathrm{mV}$ \\
\hline Entire output noise & $0.76 \mathrm{mV}$ \\
\hline CNR & $82.1 \mathrm{~dB}$ \\
\hline
\end{tabular}

\section{Measurement results}

The design of the analyzed system was focused on the demodulated sound quality, directivity and range. Actual hearing tests revealed the need for a detailed analysis of noise sources. The main difficulty of noise measurements in this particular case was the presence of the large but inaudible carrier. Spectral analyzers have a limited dynamic range, which makes it impossible to measure very small signals when large spectral components are present. To get around this problem, we performed noise measurements with the oscillator disconnected from the rest of the system. 
In order to eliminate the on-board oscillator as a possible cause of audible idle noise, the ultrasonic transmitter was tested by using a linear power amplifier and two test signals. One test signal was generated with a function generator and the other by the quadrature oscillator of the designed system. No audible noise was perceived in either case at normal carrier levels.

Measurements were performed with an HP3589A spectrum analyzer. The results of the measurements and theoretical analysis are summarized in Fig. 10. Noise power was calculated from measured power spectrum densities over the frequency band from $20 \mathrm{kHz}$ to $60 \mathrm{kHz}$. This band contains all frequencies that can modulate the amplitude of the ultrasonic carrier and demodulate into audible sound or noise.

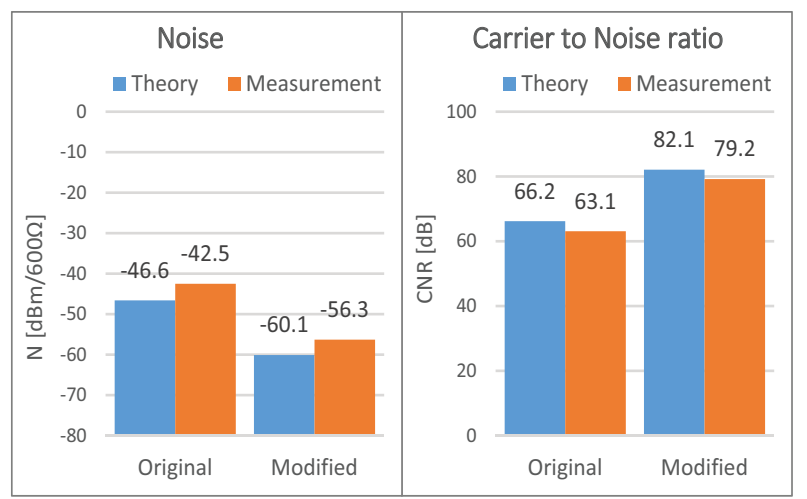

Figure 10: Theoretical and measured Noise (lower is better) and Carrier to Noise ratio (higher is better) before and after the modification.

The theoretical noise powers are lower than the measured ones in both the case of the original and the modified driver. This can be explained by the frequency response of the output filter shown in Fig. 8 which indicates that noise power density that appears on the transmitter terminals is amplified in certain frequency regions. The actual acoustic response is much more limited by the frequency response of the utilized PZT transducers.

The analysis as well as the experimental results revealed the main source of noise and enabled us to minimize its effect on the output signal without modifying the printed circuit board (PCB). This made it possible for the modification to be implemented on the existing production series. The improved version has considerably less perceptible idle noise as well as a slightly larger maximum volume.

\section{Conclusions}

The designed modulator amplifier system for a PAA resulted in good audio reproduction but exhibited noticeable acoustic noise when idle. We performed a noise analysis of the complete signal path and identified the analogue multiplier as the most significant source of the noise. This noise source is virtually independent of the signal levels, therefore higher signal levels at the multiplier input improve the output signal to noise ratio. The improvement of the device has been achieved by increasing the audio signal amplitude by a factor of 10 before it is fed into the modulator and by reducing it by approximately the same amount on the other side of the modulator. This change was possible without a PCB redesign and could therefore be implemented on the existing production series. The change has resulted in a $16 \mathrm{~dB}$ improvement in the $C N R$, which is enough to reduce the idle noise to an acceptable level.

\section{Acknowledgments}

The author would like to thank M. Ciglar of Ultrasonic d.o.o. for the financial support of the project, A. Levstek for many helpful discussions and S. Beguš for the use of an anechoic chamber.

\section{References}

1. P. J. Westervelt, "Parametric Acoustic Array," J. Acoust. Soc. Am., vol. 35, no. 4, p. 535, 1963, https://doi.org/10.1121/1.1918525

2. I. Esipov, K. Naugolnykh, and V. Timoshenko, "The Parametric Array and Long-Range Ocean Research," Acoust. Today, vol. 6, no. 2, p. 20, 2010, https://doi.org/10.1121/1.3467644

3. S. Tang, G. Zhu, J. Yin, X. Zhang, and X. Han, "A modulation method of parametric array for underwater acoustic communication," Appl. Acoust., vol. 145, pp. 305-313, Feb. 2019, https://doi.org/10.1016/j.apacoust.2018.07.032

4. J. N. Tjo/tta and S. Tjo/tta, "Theoretical study of the penetration of highly directional acoustic beams into sediments," J. Acoust. Soc. Am., vol. 69, no. 4, pp. 998-1008, Apr. 1981, https://doi.org/10.1121/1.385594

5. L. Kopp, D. Cano, E. Dubois, L. Wang, B. Smith, and R. F. W. Coates, "Potential performance of parametric communications," IEEE J. Ocean. Eng., vol. 25, no. 3, pp. 282-295, Jul. 2000, https://doi.org/10.1109/48.855259

6. H. O. Berktay, "Possible exploitation of non-linear acousticsin underwatertransmitting applications," 
J. Sound Vib., vol. 2, no. 4, pp. 435-461, Oct. 1965, https://doi.org/10.1016/0022-460X(65)90122-7

7. L. Bjørnø, "Introduction to nonlinear acoustics," Phys. Procedia, vol. 3, no. 1, pp. 5-16, Jan. 2010, https://doi.org/10.1016/j.phpro.2010.01.003

8. M. Ciglar, "Acouspade," http://www.ultrasonic-audio.com/, [Accesed: 31. 03. 2019].

9. "Audio Spotlight," https://holosonics.com/, [Accesed: 31. 03. 2019].

10. R. Haberkern, "Soundlazer," http://www.soundlazer.com/, [Accesed: 31. 03. 2019].

11. E. Hong, S. V. Krishnaswamy, C. B. Freidhoff, and S. Trolier-McKinstry, "Micromachined piezoelectric diaphragms actuated by ring shaped interdigitated transducer electrodes," Sensors Actuators A Phys., vol. 119, no. 2, pp. 521-527, Apr. 2005, https://doi.org/10.1016/j.sna.2004.10.019.

12. M. Pirc and A. Levstek, "Sources of Noise in Practical Implementations of Modulators / Amplifiers for Parametric Acoustic Arrays," in Conference 2014, proceedings / 50th International Conference on Microelectronics, Devices and Materials, October 8 - October 10, 2014, pp. 157-162.

13. W.-S. Gan, J. Yang, and T. Kamakura, "A review of parametric acoustic array in air," Appl. Acoust., vol. 73, no. 12, pp. 1211-1219, Dec. 2012, https://doi.org/10.1016/j.apacoust.2012.04.001

14. H. M. Merklinger, "Improved efficiency in the parametric transmitting array," J. Acoust. Soc. Am., vol. 58, no. 4, p. 784, 1975, https://doi.org/10.1121/1.380750

15. M. Yoneyama, "The audio spotlight: An application of nonlinear interaction of sound waves to a new type of loudspeaker design," J. Acoust. Soc. Am., vol. 73, no. 5, p. 1532, 1983, https://doi.org/10.1121/1.389414

16. J. J. (HSS) Croft and J. O. (HSS) Norris, "Theory, History, and the Advancement of Parametric Loudspeakers," 2003.
17. "Air Ultrasonic Ceramic Transducers 400ST/R160," http://www.prowave.com.tw/english/products/ ut/open-type/400s160.htm.

18. A. Levstek, "Amplitude Stabilization in Quadrature Oscillator for Low Harmonic Distortion," Inf. MIDEM, vol. 43, no. 3, pp. 185-192, 2013.

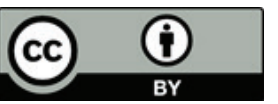

Copyright $\odot 2019$ by the Authors. This is an open access article distributed under the Creative Commons Attribution (CC BY) License (https://creativecommons.org/licenses/by/4.0/), which permits unrestricted use, distribution, and reproduction in any medium, provided the original work is properly cited.

Arrived: 31. 03. 2019

Accepted: 15. 04. 2019 